\title{
Energy Resolution of Xenon Proportional Counters: Monte Carlo Simulation and Experimental Results
}

\author{
P. J. B. M. Rachinhas, T. H. V. T. Dias, A. D. Stauffer, F. P. Santos, and C. A. N. Conde
}

\begin{abstract}
The gas multiplication factor $M$ and energy resolution $R$ of xenon gas cylindrical proportional counters are investigated experimentally and calculated theoretically using a Monte Carlo technique to simulate the growth of singleelectron-initiated avalanches. A good agreement is found between calculated and experimental data.

The experimental and the calculated results are presented as a function of the reduced voltage $K$ and the reduced anode radius $N a$, where $N$ is the number density and $a$ the anode radius. The Monte Carlo results for the intrinsic energy resolution $R_{\text {int }}$ are discussed in terms of the parameter $f$ which characterizes the statistical fluctuations of the avalanche gains.

The present calculations have revealed that there is an intrinsic dependence of $f$ on the critical value $S_{c}$ of the reduced electric field at the onset of multiplication. This has enabled the parameterization of $f$ and of the intrinsic energy resolution $R_{\text {int }}$ in terms of the reduced voltage $K$ only and has explained why, for a given range of operating values for $M$, energy resolution in a cylindrical proportional counter is improved for thinner anode wires and lower pressures.
\end{abstract}

\section{INTRODUCTION}

G AS-FILLED proportional counters have been used for more than four decades in various areas of experimental physics as well as in other areas of science and technology, such as in environmental studies, the mining industry, medicine, etc., where the detection of ionizing radiations is required [1].

The understanding of the working mechanisms involved in this type of radiation detector has been the subject of a continued research effort, and its main characteristics-the energy resolution $R$ and gas multiplication factor $M$-have been thoroughly investigated [2]-[12].

The energy resolution depends upon many technical factors, like anode wire nonuniformity, attachment of electrons to impurities, and amplifier noise, whose control can keep the value of $R$ to a minimum [5]. There are intrinsic factors, however, that put an ultimate limit on the achievable energy resolution [3]-[6], such as the statistical fluctuations in the

Manuscript received January 23, 1996; revised May 14, 1996. This work was supported by JNICT (Junta Nacional de Investigação Científica e Tecnológica) through Project PEDIC/SIS/1853/94. The work of P. J. B. M. Rachinhas was supported by JNICT Grant BM-2795/92. The work of T. H. V. T. Dias was supported by a travel grant by Fundação Calouste Gulbenkian-Lisboa. This work was presented at the 1995 IEEE Nuclear Science Symposium, October 21-28, 1995, San Francisco, CA.

P. J. B. M. Rachinhas, T. H. V. T. Dias, F. P. Santos, and C. A. N. Conde are with the Departamento de Física, Universidade de Coimbra, P-3000 Coimbra, Portugal.

A. D. Stauffer is with the Department of Physics and Astronomy, York University, Toronto, M3J 1P3 Canada.

Publisher Item Identifier S 0018-9499(96)06198-9. number of primary electrons (characterized by the so-called Fano factor, $F$ ), the mean energy to produce a primary electron, $w$, and the statistical fluctuations, $\sigma_{A}$, in the gain $A$ (number of electrons at the anode) of each avalanche initiated by a single primary electron. These fluctuations are characterized by the parameter $f$, defined as

$$
f=\frac{\sigma_{A}^{2}}{M^{2}}
$$

where $M$, the multiplication factor, is the mean value of the single-electron avalanche gain $A$. For an ionizing radiation of energy $E_{x}$, the intrinsic energy resolution $R_{\text {int }}$ of a proportional counter-a theoretical lower limit to the achievable experimental energy resolution-is given in terms of the parameters $F, f$, and $w$ by [1]

$$
R_{\text {int }}(\%)=235 \sqrt{\frac{(F+f) w}{E_{x}}} .
$$

The behavior of the parameters $F$ and $w$ with incident radiation energy is characteristic of the gas and has been investigated in earlier work for $\mathrm{X}$ rays in xenon using Monte Carlo simulation [13]-[16]. The statistical fluctuations parameter $f$, which depends not only on the gas but also on the operational conditions of the detector, is the dominant factor in the energy resolution of proportional counters and is the subject of this paper.

The purpose of the present work is to describe and discuss in detail the factors which affect the energy resolution in xenon gas cylindrical proportional counters, using Monte Carlo simulation together with experimental measurements. It will be shown that the dependence of $f$-and hence of $R$-on the multiplication factor $M$, anode wire radius $a$, and pressure $p$ is inherently connected with the critical reduced electric field $S_{c}=(E / N)_{c}$ defined as the ratio, at the onset of multiplication, of the electric field $E$ to the gas number density $N$. A dependency of $S_{c}, f$, and $R$ on the reduced anode voltage $K=V / \ln (c / a)$ is found, where $V$ is the applied voltage and $c / a$ is the cathode-to-anode radius ratio.

The Monte Carlo simulation method is summarized in Section II, and the experimental setup is described in Section III. Monte Carlo calculated and measured experimental results will be presented and discussed in Section IV.

The range of operation investigated in this work comprises multiplication factors from $M \sim 10$ to $M \sim 10000$.

Two different anode wire radii are considered in the experiments and in the calculations. Experimental results were always taken at 760 Torr, but Monte Carlo simulation data 
TABLE I.

$\mathrm{Na}$ Values Used in this Work aNd CoRresponding Anode Radius and Pressure of the Gas at $T=20^{\circ} \mathrm{C}$

\begin{tabular}{ccccc}
\hline $\begin{array}{c}N a \\
\left(10^{17} \mathrm{~cm}^{-2}\right)\end{array}$ & 0.066 & $\begin{array}{c}0.250 \\
\bullet \mathrm{MC} \\
\Delta \mathrm{Exp}\end{array}$ & $\begin{array}{c}0.626 \\
\square \mathrm{MC}\end{array}$ & $\begin{array}{c}1.190 \\
\mathbf{M C} \\
0 \mathrm{Exp}\end{array}$ \\
\hline$a(\mu \mathrm{m})$ & 10 & 10 & 47.5 & 47.5 \\
$p$ (Torr) & 200 & 760 & 400 & 760 \\
\hline
\end{tabular}

includes calculations at two lower pressures as well. The corresponding values for the reduced anode radius, $N a$, are listed in Table I.

As indicated in Table I, throughout this work the symbols $\circ, \bullet, \square$, and $\mathbf{\square}$ will be used to distinguish the Monte Carlo data at the four different $N a$ values, and the symbols $\triangle$ and $\diamond$ are reserved for the experimental data, taken at two of these $\mathrm{Na}$ values. A series of points with same symbol will correspond to results for a range of values of the multiplication factor $M$.

\section{Monte Carlo Method}

Single-electron-initiated avalanches in the cylindrically symmetric electric field of a proportional counter were simulated by a Monte Carlo technique, following all electrons individually to the anode along their various paths determined by multiple elastic and inelastic collisions in the gas. The electron scattering cross sections in xenon used in this work have been described in earlier publications [15].

A null collision technique was used to determine the time of flight of the electrons between successive collisions with the background neutral gas atoms. Between collisions, the equations of motion of the electrons are integrated in sixdimensional phase space by the fast "leap-frog" numerical method [17]. The type of collision-elastic, excitation, or ionization-is decided according to the corresponding integral scattering cross sections. When excitation occurs, partial excitation cross sections are used to decide among 13 excited states. The higher excited state is allowed to undergo Hornbeck-Molnar ionization according to a pressure dependent probability [18]. The angular distribution of the electrons scattered in elastic and excitation collisions is determined by angular-differential cross sections. For ionizing collisions, isotropy is assumed for the emission of the two electrons, and the energy partition between them follows an energydifferential cross section.

Space charge effects are neglected, as well as secondary processes in the gas such as electron recombination or attachment to the gas molecules.

All the Monte Carlo calculated results presented in this work were obtained by averaging over a sample of $\sim 5000$ singleelectron-initiated avalanches. Single electrons were released at a radial position $r_{0}$ such that $S\left(r_{0}\right)=15 \mathrm{Td}^{1}$ (a value below the multiplication threshold under uniform field condition),

${ }^{1} 1 \mathrm{Td}=10^{-17} \mathrm{Vcm}^{2}$.

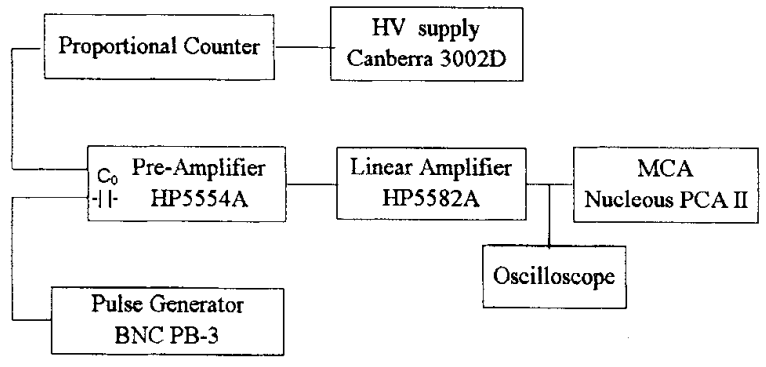

Fig. 1. Block diagram of the experimental setup.

with an energy randomly chosen between 0 and $5 \mathrm{eV}$ and an isotropic angular distribution.

The errors for the calculated results are estimated to be at most $2 \%$ for the multiplication factor $M$ and reduced critical field $S_{c}$ and $4 \%$ for the statistical fluctuations parameter $f$.

In an earlier paper [19], preliminary results for singleelectron-initiated avalanches simulated by a Monte Carlo method were presented. The present model, however, leads to a much better agreement with the experimentally measured multiplication factors. A full description will be included in a later publication.

\section{EXPERIMENTAL SYSTEM}

The experimental results described in this work were obtained with a 20 -cm-long stainless steel cylindrical proportional counter with an inner radius of $2.55 \mathrm{~cm}$ and a tungsten anode wire. The measurements were made using 10 and 47.5 $\mu \mathrm{m}$ radius anode wires, with the smaller gauge being gold coated. The counter was filled with pure xenon (N45) at 760 Torr, $T=20^{\circ} \mathrm{C}$. The gas was continuously purified with SAES ST707 getters. The entrance window was cut at the midpoint of the cathode and sealed with a $25-\mu$ m-thick Kapton film. In all measurements, the incident x-ray energy was $E_{x}=5.9 \mathrm{keV}$, the $K_{\alpha}$-fluorescence $\mathrm{x}$-rays from a ${ }^{55} \mathrm{Fe}$ radioactive source ( $\sim 200$ counts/s), whose $K_{\beta}$-line was filtered with a Cr foil.

The experimental setup is schematically shown in Fig. 1. To enable the monitoring of the absolute charge gain during anode signal data acquisition, a signal of known amplitude from a pulse generator model BNC PB-3 is fed into the test input of a HP5554A charge-sensitive preamplifier through a $C_{0}=1.0 \mathrm{pF}$ precision capacitor. The output from the preamplifier goes into a linear amplifier of the type HP5582A, where the integration and differentiation time constants were set at $5 \mu \mathrm{s}$ for all measurements. The signals proceeding from the proportional counter and from the pulse generator were recorded simultaneously in a Nucleus PCA II multichannel analyzer. The centroid position, peak amplitudes, and energy resolution of the measured pulse-height distributions were obtained by fitting these distributions with a Gaussian function superimposed on a linear background.

\section{RESULTS AND DISCUSSION}

Under a uniform electric field $E^{0}$, a swarm of electrons can, in principle, reach a state of hydrodynamical equilibrium 


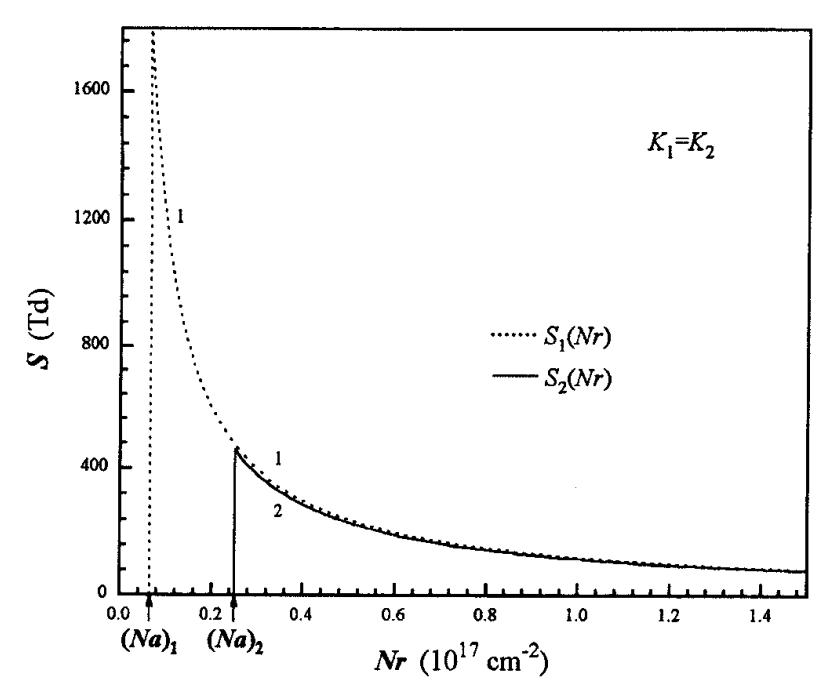

Fig. 2. Reduced electric field $S$ as a function of reduced radial distance $N r$, for a given $K$ and two $N a$ values $\left((N a)_{1}=.066,(N a)_{2}=.250 \times 10^{17} \mathrm{~cm}^{-2}, K=115\right.$ Volt $)$.

in the gas, which can be described by the value of the reduced electric field $S^{0}=E^{0} / N$ only.

In the cylindrical geometry of a proportional counter, however, where the reduced electric field $S(N r)=E(r) / N$ has a spatial variation given by

$$
S=\frac{V}{\ln (c / a)} \frac{1}{N r}=K \frac{1}{N r} \quad \text { with } \quad K=\frac{V}{\ln (c / a)}
$$

$S$ can vary rapidly with the reduced radial distance $N r$, especially when $N r$ is small, and the electrons are observed to lag behind the equilibrium condition- a lag connected to the electric field gradient. Therefore, the state of the electrons must now be described not only by the value of $S$, but also by the reduced field gradient, $\operatorname{grad} S=\partial S / \partial(N r)=-K /(N r)^{2}=$ $-S^{2} / K$ or, equivalently, the local state at any reduced radial position $N r$ can be described in terms of the reduced anode voltage $K$ only [9], [20].

We wish to emphasize the fact that the operational parameter $K$ fully characterizes the way an avalanche develops toward the anode within the cylindrical proportional counter, since for a given $K$, the reduced field $S$ and its gradient are uniquely defined as a function of the reduced radial distance $N r$, from $N c$ to $N a$. For a fixed $K$, the extension of an avalanche will be controlled by the magnitude of the reduced anode radius $N a$, as this value determines the reduced radial distances that the electrons must travel to reach the anode. This is illustrated in Fig. 2, where two partially coincident $S(\mathrm{Nr})$ curves-corresponding to two distinct $\mathrm{Na}$ values but the same $K$-are depicted.

From the above, we can assert that any physical quantity defineable in the cylindrical proportional counter will be uniquely specified by the two operational variables $K$ and $N a$, i.e., by the reduced anode voltage and radius. Locally defined quantities, such as the reduced electric field $S_{c}$ at the onset of multiplication, are expected to depend on $K$ only, while quantities whose value depends on the entire development of the avalanche, such as the global results $M$ or $f$, are expected to depend not only on $K$ but also on $N a$.

Throughout this section, we will be describing our Monte Carlo simulation results for the multiplication factor $M$, the critical reduced electric field $S_{c}$, the statistical fluctuations parameter $f$, and the energy resolution $R$, discussing these quantities in terms of the reduced anode voltage $K$ and radius $N a$.

Experimental results for $M$ and $R$ will also be presented and compared with the calculated data.

As will be seen later, an important result of the present simulation is that, for the $M$ range investigated in this work, the statistical fluctuations parameter $f$ appears to be independent of $N a$, i.e., $f$ can be characterized by $K$ only.

\section{A. Multiplication Factor $M$}

The results obtained experimentally for the multiplication factor $M$ with the proportional counter described in the previous section are shown in Fig. 3 as a function of the reduced anode voltage $K$. These $M$ values are obtained from

$$
M=\frac{Q}{e E_{x} / w}
$$

where $Q$ is the mean value of the charge collected at the anode and $e$ is the charge of the electron. Note that $n=E_{x} / w$ is the number of primary electrons produced per absorbed $\mathrm{X}$ ray photon. To obtain the value of $Q$, the channel number $m$ corresponding to the centroid position of each $5.9 \mathrm{keV}$ pulseheight distribution was converted to the equivalent charge $Q$ through

$$
Q=C_{0} V_{0} \frac{\zeta_{0} m}{\zeta m_{0}}
$$

where $V_{0}$ is the amplitude of the signal from the pulse generator and $m_{0}$ is the corresponding channel number in the multichannel analyzer. The factors $\zeta$ and $\zeta_{0}$ were introduced to correct the amplitude of the signals from the proportional counter and the pulse generator for the attenuation effect caused by the finite magnitude of the linear amplifier time constant [7], [9], [21]-[23], using the value $0.45 \mathrm{~cm}^{2} \mathrm{~V}^{-1} \mathrm{~s}^{-1}$ for the ion mobility [22]. A value of $w=22.4 \mathrm{eV}$ [14], [15] was assumed at $5.9 \mathrm{keV}$ and used in (4). An error of $\sim 5 \%$ is estimated for the experimental $M$ values shown in Fig. 3, with the main contribution arising from the charge calibration procedure. In addition, we estimate a systematic contribution of $\sim 3 \%$, related to the choice of $w$ in (4). In fact, the calculated $w$-value is expected to become larger if the possibility of $\mathrm{Xe}$ inner-shell electron-impact ionization together with associative and transfer ionization processes is introduced into our simulation model which yields values for $w$ [18], [24], [25].

The Monte Carlo values for the multiplication factor $M$ are also included in Fig. 3.

Both the experimental and the calculated results show that $M$ is an increasing function of $K$ for any $N a$ value. Where comparisons can be made (i.e., at $N a=0.250$ and $1.190 \times 10^{17} \mathrm{~cm}^{-2}$ ), a good agreement is observed, especially for the $10 \mu \mathrm{m}$ anode, although in both cases the agreement is 


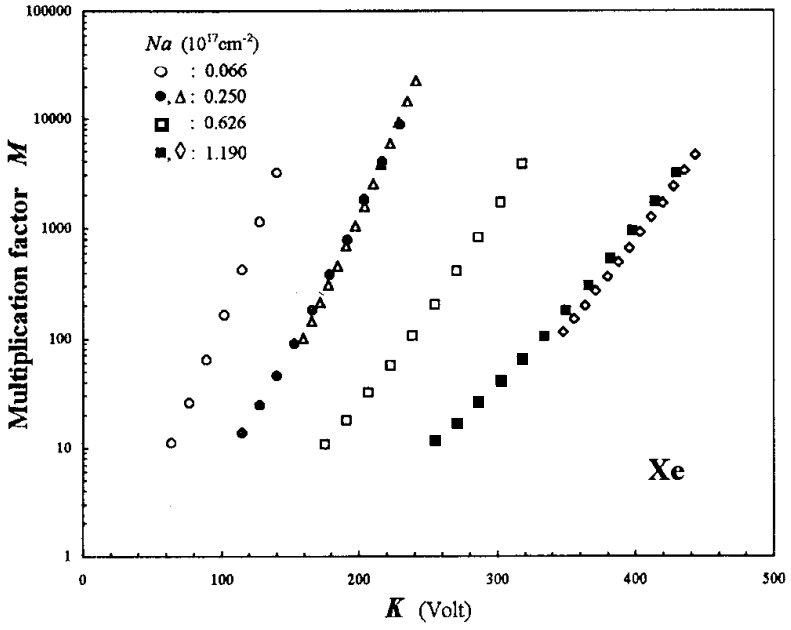

Fig. 3. Experimental $(\triangle, \diamond)$ and Monte Carlo simulation $(\diamond, \bullet, \square, \boldsymbol{\square})$ results for the multiplication factor $M$ as a function of reduced anode voltage $K$, for different reduced anode radii $N a$.

poorer for the lower $M$ values. We think this reflects the effect of the lower signal-to-noise ratio in this region, as the observed discrepancies are not likely to be related to secondary effects such as recombination - a negligible effect at the pressure used - or space charge - which according to an analysis based on the work of [26] and [27] would cause a gain shift lower than $2 \%$ for our counting rate and gas amplification range.

We must emphasize that, with $M$ being a global quantity, it depends on $K$ and on $N a$. Note that the different $M$ values obtained for a given $K$ reflect the amount of multiplication occurring in the different reduced distances traveled by the electrons to reach the anode as the reduced anode radius $\mathrm{Na}$ varies; see Fig. 2.

\section{B. Critical Reduced Electric Field $S_{c}$ at the Onset of Multiplication}

In Fig. 4 , the Monte Carlo $S_{c}$ values are represented as a function of the reduced anode voltage $K$, for all four reduced anode radius $N a$.

As it was expected, we verify that $S_{c}$ depends on $K$ but not on $N a$, since all points, irrespective of their $N a$ value, are joined in one common curve. Moreover it is observed that $S_{c}$ decreases with increasing $K$.

We will now try to clarify why $S_{c}$ is a decreasing function of $K$.

For that purpose we will make use of the specific example in Fig. 5, which portrays two situations taken from the Monte Carlo results at $\mathrm{Na}=1.190 \times 10^{17} \mathrm{~cm}^{-2}$ (series represented by symbol $\mathbf{0})$. The full curves 1 and 2 represent $S(N r)$ for two $K$ values, with $K_{2}<K_{1}$. The calculated $S_{c}$ values belonging to each $S(N r)$ curve are pointed out, and we see that $S_{c 2}>S_{c 1}$, in conformity with Fig. 4 . The dotted curves represent the corresponding gradients $\operatorname{grad} S(\mathrm{Nr})$.

If we look into the grad $S$ curves, we verify that for any given $S$ value in the two $S(N r)$ curves, we have $(\operatorname{grad} S)_{2} /(\operatorname{grad} S)_{1}=K_{1} / K_{2}>1$, as exemplified by the vertical dotted lines. As a larger value of field gradient will

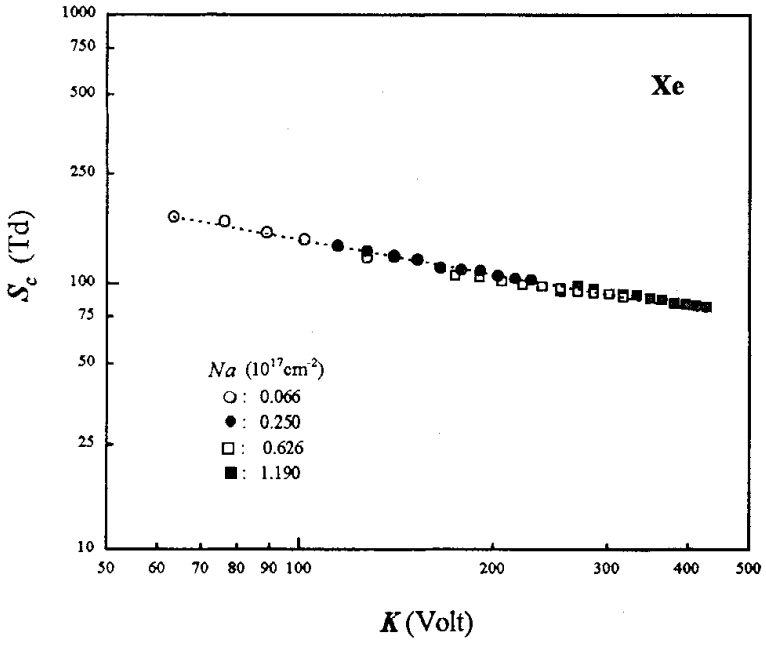

Fig. 4. Monte Carlo simulation results for the reduced critical electric field $S_{C}$ as a function of the reduced anode voltage $K$, for different reduced anode radii $N a$.

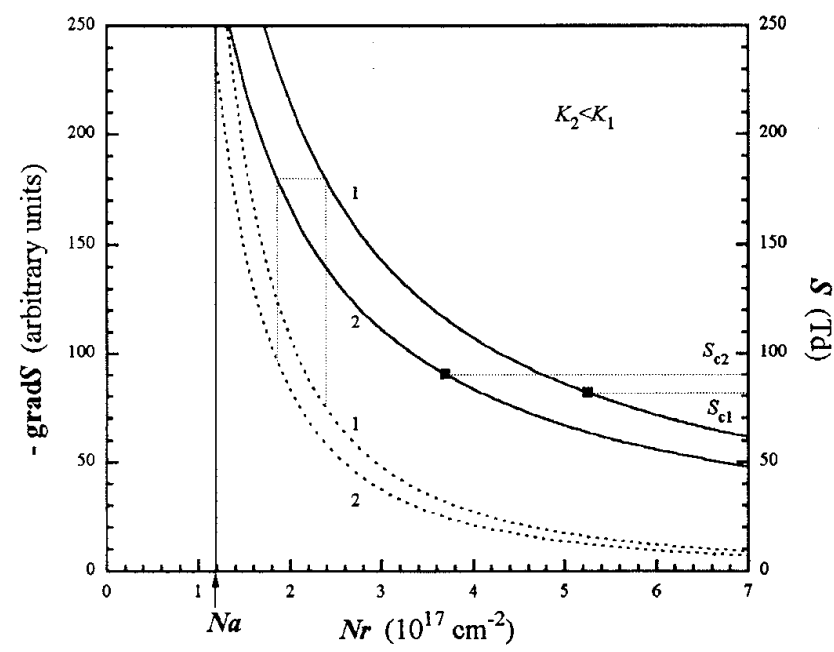

Fig. 5. Reduced electric field $S(-)$ and magnitude of $\operatorname{grad} S(\ldots)$ for a fixed reduced anode radius $N a$ and two distinct $K$ values $\left(\mathrm{Na}=1.19010^{17} \mathrm{~cm}^{-2}, K_{1}=429.5, K_{2}=334.1\right.$ Volt $)$, as a function of reduced radial distance $N r$. The Monte Carlo value for the reduced critical field $S_{C}$ for each situation is pointed out on the two $S(N r)$ curves.

always be associated with a wider lag behind the equilibrium for the electrons, the onset of multiplication will naturally occur later in the field for the case of the $S(N r)$ curve with the lower $K$. Thus it is understandable that $S_{c 2}>S_{c 1}$ and that $S_{c}$ is a decreasing function of the reduced anode voltage $K$.

\section{Statistical Fluctuations Parameter $f$}

In Fig. 6, we represent the Monte Carlo values for parameter $f$ as a function of $S_{c}$ for the four $N a$ values investigated.

We observe that the higher fluctuations $f$ are associated with the lower $S_{c}$ values. Furthermore, Fig. 6 reveals that all data points fall on a common curve. This important result indicates that, for the $S_{c}$ range investigated (lower than $\sim 200 \mathrm{Td}$ ), the statistical fluctuations parameter $f$ appears to be controlled 


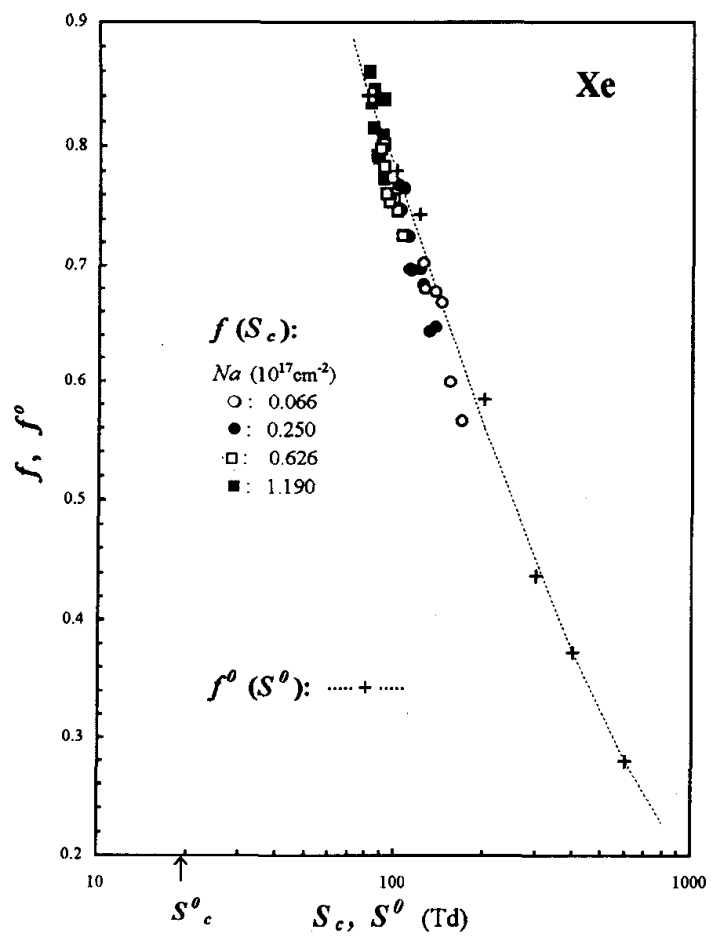

Fig. 6. Monte Carlo values $f$ and $f^{0}$ for the statistical fluctuations parameter of the avalanche gain: i) $f(0, \bullet, \square$, and $\boldsymbol{\square})$ values were calculated in cylindrical field geometry and are represented as a function of the critical reduced electric field $S_{c}$; ii) $f^{0}(+)$ were calculated in uniform field geometry and are represented as a function of the reduced uniform electric field $S^{0}$ (the dotted line is a simple guide to the eye). The value $S_{c}^{0} \sim 18 \mathrm{Td}$ indicated by an arrow is the critical reduced field for the uniform field geometry [28].

by the first stages of electron multiplication only. This is ascertained by the resemblance of $f\left(S_{c}\right)$ with the behavior of the statistical fluctuations $f^{0}\left(S^{0}\right)$ calculated for a uniform electric field $S^{0}$; see again Fig. 6 . Note that $S_{c}$ is always higher than $S_{c}^{0}$, the onset of multiplication for the case of uniform field, also marked in Fig. 6.

Recalling that, as we stated before, $S_{c}$ is characteristic of a given $K$ in a cylindrical field geometry, we reach the conclusion that, for the $K$ and $N a$ ranges investigated, $f$ will also be uniquely defined for a given $K$ value, i.e., although $f$ is a global quantity, it will be independent of the $N a$ value.

This is verified in Fig. 7, where we plot the Monte Carlo $f$ results as a function of the reduced anode voltage $K$, for the four $N a$ values. This figure shows indeed that all the calculated $f$ data points appear joined in one common curve, i.e., $f$ is found to depend on anode voltage $K$ only.

Moreover, we see that $f$ is an increasing function of $K$. This behavior of $f$ is easy to justify, if we recall that our calculated results have shown that higher fluctuations are associated with lower $S_{c}$ values (see Fig. 6) and that in turn a lower $S_{c}$ was found to be associated with a higher $K$ (see Fig. 4).

\section{Energy Resolution $R$}

In Fig. 8 the intrinsic energy resolution $R_{\text {int }}$ at $5.9 \mathrm{keV}$ calculated via our Monte Carlo simulation is represented as a function of reduced anode voltage $K$, for the usual four $N a$

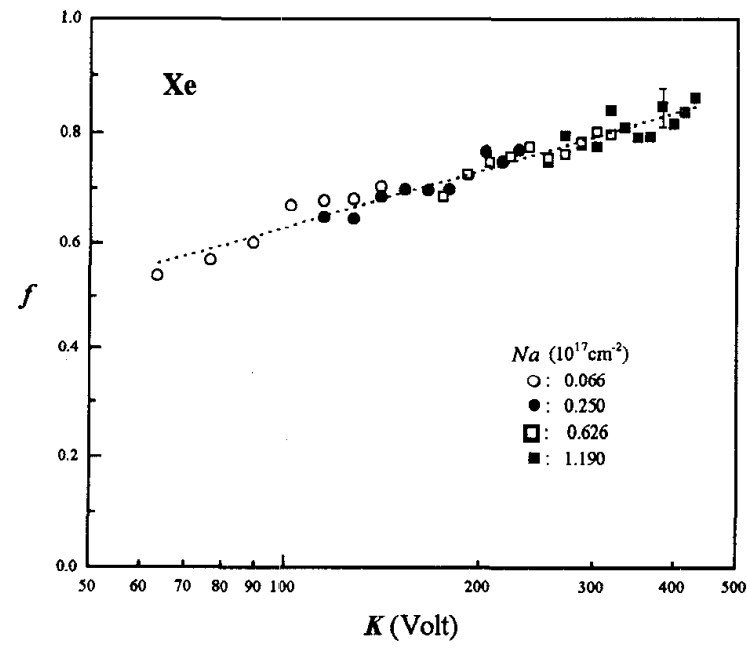

Fig. 7. Monte Carlo simulation results for the statistical fluctuations parameter $f$ of the avalanche gain as a function of reduced anode voltage $K$, for different reduced anode radii $\mathrm{Na}$.

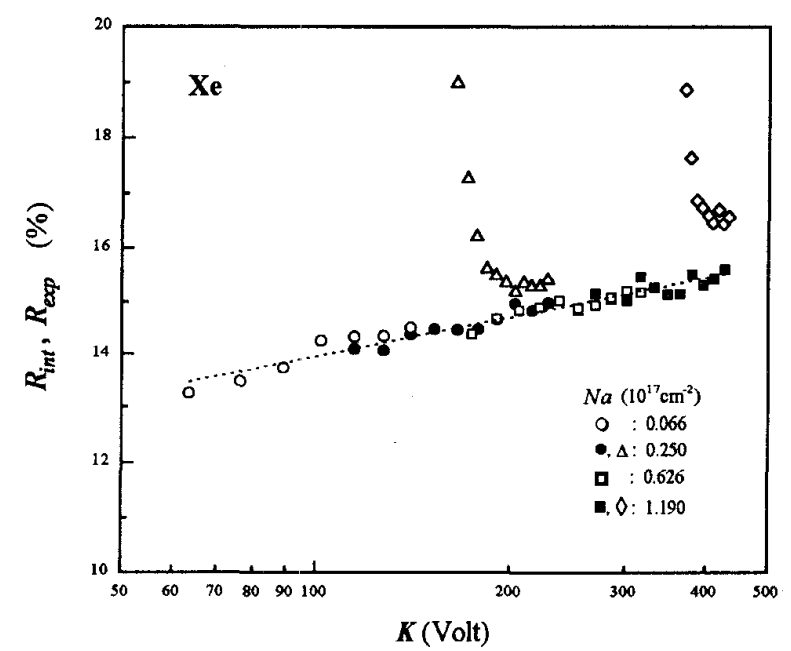

Fig. 8. Experimental values for the energy resolution $R_{\exp }(\triangle, \diamond)$ and Monte Carlo simulation results for the intrinsic energy resolution $R_{\text {int }}(0, \bullet, \square, \square)$ as a function of the reduced anode voltage $K$, for different reduced anode radii $N a$ and for an x-ray energy $E_{x}=5.9 \mathrm{keV}$.

values. $R_{\text {int }}$ was deduced from (2) using the values $F=0.3$ and $w=22.4 \mathrm{eV}$ [14], [15] together with the results for $f(K)$ shown in Fig. 7.

Fig. 8 includes as well the experimental results for the energy resolution $R_{\exp }$, which were measured at two of the $\mathrm{Na}$ values with the experimental system described in Section III.

We see that in general $R_{\text {int }}$ deteriorates as $K$ increases, a behavior which directly reflects the increase in the calculated $f(K)$ results shown in Fig. 7 .

The experimental energy resolution $R_{\text {exp }}$ exhibits a similar tendency as $K$ increases and approaches to within $\sim 5 \%$ and $\sim 8 \%$ of the calculated values for $R_{\text {int }}$, at the corresponding $N a$ values. The degradation perceived for the thicker anode is possibly due to the poorer surface finishing of this nongold-coated anode wire (a rough calculation indicates that a variation of $\sim 0.5 \%$ in the anode radius will cause a 
$\sim 3 \%$ variation in gain, which is expected to degrade $R_{\text {exp }}$ appreciably [5], [29]). On the other hand, we also expect an improved agreement between $R_{\exp }$ and $R_{\text {int }}$, once revised $w$-values are available from our more elaborate simulation planned for the near future.

As $K$ becomes lower in each of the two series of the experimental results, the accuracy of the measured values of $R_{\exp }$ deteriorates due to the broadening of the pulse-height distributions by the effect of low signal-to-noise ratios. This is illustrated in Fig. 8 by the increasing deviation of $R_{\exp }$ from the approximate straight line behavior for the intrinsic resolution predicted by our simulated results.

Recalling that $M$ increases with $K$ for each $N a$ value (see Fig. 3), Fig. 8 also shows that energy resolution tends to deteriorate with increasing $M$, a behavior commonly observed in experimental work with proportional counters [1], [6].

Finally, we observe in Fig. 8 that the four series of calculated data points—symbols $\circ, \bullet \square, \mathbf{\square}$-appear in line from left to right in a sequence which corresponds to increasing $\mathrm{Na}$. In fact, as can be seen from Fig. 3, for a fixed value of $M$ the smaller values of $N a$ correspond to smaller values of $K$ and hence of $R$. This behavior, which is obviously connected with the behavior of $f(K)$ in Fig. 7, establishes the justification for the experimentally observed effect that the energy resolution for a given $M$ operating value is improved when lower pressures or/and thinner anode wires are used in a cylindrical proportional counter, i.e., when $N a$ is lowered. This effect was verified in our experimental results-symbols $\triangle, \diamond$-taken at 760 Torr, which show a better energy resolution for the thinner anode wire. Likewise, an improvement of $R$ with a decrease of pressure and anode wire radius has been observed by [10] and [11] for a $22 \mathrm{keV} \mathrm{X}$-ray energy using a square section Xe proportional counter. The same effect was predicted for the case of Ar by [2] and [3].

Besides the technical limits imposed on the achievable minimum $N a$ reduced anode radius, however, the ultimate energy resolution is expected to be also inherently limited by some lower bound on the parameter $f$. In our Monte Carlo calculations under uniform electric field, to be discussed in a later publication, this limit was found to be $f^{0} \sim 0.2$.

Moreover, at low enough $\mathrm{Na}$ values, electrons can spiral significantly around the anode before being collected, as we have already observed with the present Monte Carlo model (below $N a \sim 0.025 \times 10^{17} \mathrm{~cm}^{-2}$ ), and although this effect was not investigated in the present work, it may have some influence on energy resolution.

We plan to extend our work in the future to the promising field of microstrip gas detectors, investigating detectors with smaller $N a$ values as well as different gas fillings, such as Penning mixtures.

\section{CONCLUSIONS}

We have shown that the detailed Monte Carlo simulation model we developed produces very good predictions for the multiplication factor, $M$, and the energy resolution, $R$, in xenon-filled cylindrical proportional counters. A good agreement was found between the calculated and the experimental results for $M$, and it was found that the experimental energy resolution at $5.9 \mathrm{keV}$ measured at 760 Torr for 10 and 47.5 $\mu \mathrm{m}$ anode radii approaches very closely the calculated intrinsic values.

The intrinsic energy resolution is discussed in terms of the parameter $f$ which describes the statistical fluctuations in the number of electrons in single-electron-initiated avalanches. The parameter $f$ is calculated using a Monte Carlo simulation of the growth of avalanches in xenon for a cylindrical electric field geometry.

It was found that the dependence of $f$ on the currently used variables-multiplication factor, anode wire radius, and gas pressure-is intrinsically connected with the critical reduced electric field $S_{c}$ defined at the onset of multiplication.

The Monte Carlo results for $S_{c}$ and $f$ have shown that these are intrinsic characteristics of the development of an electron avalanche, i.e., of the reduced anode voltage $K=V / \ln (c / a)$, and a dependence of $S_{c}$ and $f$ on $K$ was demonstrated. The experimentally observed dependence of energy resolution on pressure and anode wire radius was explained in terms of the behavior of $f(K)$.

$S_{c}(K)$ and $f(K)$ do not in principle depend on the X-ray energy absorbed in the detector, but are expected to change with the nature of the gas used in the detector. Although the calculated intrinsic energy resolution was shown for 5.9 $\mathrm{keV} \mathrm{X}$ rays only (to compare with the experimental results), it can be estimated for other X-ray energies using (2), if the presently reported Monte Carlo values for $f(K)$ in xenon are used together with the Fano factor $F$ and the $w$ values at the required energy and for the same gas [14], [15].

\section{ACKNOWLEDGMENT}

The authors would like to acknowledge the help in computing facilities which was granted by LIP Coimbra and by the Theoretical Physics group, University of Coimbra.

\section{REFERENCES}

[1] G. F. Knoll, Radiation Detection and Measurement, 2nd ed. New York: Wiley, 1989, pp. 161-195.

[2] G. D. Alkhazov, "Mean value and variance of gas amplification in proportional counters," Nucl. Instrum. Meth., vol. 75, pp. 161-162, 1969.

[3] - "Statistics of electron avalanches and ultimate resolution of proportional counters," Nucl. Instrum. Meth., vol. 89, pp. 155-165, 1970.

[4] J. Byrne, "Statistics of the electron multiplication process in proportional counters," Proc. Roy. Soc. Edinburgh, vol. A66, pp. 33-41, 1962.

[5] M. W. Charles and B. A. Cooke, "Proportional counter resolution," Nucl. Instrum. Meth., vol. 61, pp. 31-36, 1968.

[6] H. Sipila, "Energy resolution of the proportional counter," Nucl. Instrum. Meth., vol. 133, pp. 251-252, 1976.

[7] M. W. Charles, "Gas gain measurements in proportional counters," $J$. Phys. E: Sci. Instrum. vol. 5, pp. 95-100, 1972.

[8] T. Aoyama, "Generalized gas gain formula for proportional counters," Nucl. Instrum. Meth. Phys. Res. A Accel. Spectrum. Detect. Assoc. Equip., vol. 234, pp. $125-131,1985$.

[9] S. P. Beingessner, R. K. Carnegie, and C. K. Hargrove, "An extended parametrization of gas amplification in proportional wire chambers," Nucl. Instrum. Meth. Phys. Res. A Accel. Spectrum. Detect. Assoc. Equip., vol. 260 , pp. $210-220,1987$.

[10] H. Sakurai, B. D. Ramsey, and M. C. Weisskopf, "High pressure xenon proportional counter up to $10 \mathrm{~atm}$," Nucl. Instrum. Meth. Phys. Res. A Accel. Spectrum. Detect. Assoc. Equip., vol. 307, pp. 504-511, 1991. 
[11] H. Sakurai and B. D. Ramsey, "Dependence of energy resolution on anode diameter in xenon proportional counters," Nucl. Instrum. Meth. Phys. Res. A Accel. Spectrum. Detect. Assoc. Equip., vol. 313, pp. $155-160,1992$.

[12] I. K. Bronic,, "A study of argon-isobutane mixtures in a proportional counter: Gas amplification, w-value, and energy resolution," Radiat. Prot. Dosim, to be published.

[13] T. H. V. T. Dias, F. P. Santos, A. D. Stauffer, and C. A. N. Conde, "The Fano factor in gaseous xenon: A Monte Carlo calculation for x-rays in the 0.1 to $25 \mathrm{keV}$ energy range," Nucl. Instrum. and Meth. Phys. Res A Accel. Spectrum. Detect. Assoc. Equip., vol. 307, pp. 341-346, 1991.

[14] F. P. Santos, T. H. V. T. Dias, A. D. Stauffer, and C. A. N. Conde, "Variation of energy linearity and $w$-value in gaseous xenon radiation detectors for X-rays in the 0.1 to $25 \mathrm{keV}$ energy range: A Monte Carlo simulation study," Nucl. Instrum. and Meth. in Phys. Res. A Accel. Spectrum. Detect. Assoc. Equip., vol. 307, pp. 347-352, 1991.

[15] T. H. V. T. Dias, F. P. Santos, A. D. Stauffer, and C. A. N. Conde, "A Monte Carlo simulation of $\mathrm{x}$-ray absorption and electron drift in gaseous xenon," Phys. Rev., vol. A48, pp. 2887-2902, 1993.

[16] T. H. V. T. Dias, F. P. Santos, J. A. M. Lopes, J. F. C. A. Veloso, P. J. B. M. Rachinhas, R. E. Morgado, A. D. Stauffer, and C. A. N. Conde, "The response of xenon $\mathrm{x}$-ray detectors to full-energy absorption and fluorescence-escape events: measurement and modeling," to be published in IEEE Trans. Nucl. Sci., vol. 43, 1996.

[17] D. Potter, Computational Physics. New York: Wiley, 1973, pp. 32-34.

[18] J. A. Hornbeck and J. P. Molnar, "Mass spectrometric studies of molecular ions in the noble gases," Phys. Rev., vol. 84, pp. 621-625, 1951.

[19] P. J. B. M. Rachinhas, T. H. V. T. Dias, F. P. Santos, A. D. Stauffer and C. A. N. Conde, "Monte Carlo simulation of xenon filled cylindrical proportional counters," IEEE Trans. Nucl. Sci., vol. 41, pp. 984-988, 1994.
[20] P. Segur, A. Alkaa, S. Pineau, A. Zahraoui, A. Chouki, C. Moutarde, and S. Laffont, "Kinetic description of electrons in inhomogeneous electric fields," Plasma Sources Sci. Technol., vol. 4, pp. 183-199, 1995.

[21] E. Mathieson and M. W. Charles, "Attenuation of proportional counter pulses by equal integrating and differentiating time constants I," $\mathrm{NuCl}$. Instrum. Meth., vol. 72, pp. 155-156, 1969.

[22] R. Gott and M. W. Charles, "Attenuation of proportional counter pulses by equal integrating and differentiating time constants II," Nucl. Instrum. Meth., vol. 72, pp. 157-160, 1969.

[23] B. Breyer and M. Cimerman, "Attenuation of proportional counter pulses by pulse shaping networks," Nucl. Instrum. Meth., vol. 92, pp. 19-20, 1971.

[24] F. P. Santos, T. H. V. T. Dias, P. J. B. M. Rachinhas, R. E. Morgado, and C. A. N. Conde, "The Fano factor and $w$-value for X-rays in xenon near the $L$ and $K$ edges, allowing for electron impact inner-shell ionization," in Proc. 12th. Europhysics Sectional Conference on the Atomic and Molecular Physics of Ionized Gases, Leeuwenhorst, Noordwijkerthout, Holland, Aug. 23-26 1994, pp. 71-72.

[25] H. Cederquist, C. Biedermann, N. Selberg, E. Beebe, M. Pajek, and A. Bárány, "Strong onset of ionization in slow $\mathrm{Xe}^{q+}-\mathrm{Xe}$ collisions at very high q," Phys. Rev., vol. A47, pp. R4551-R4554, 1993.

[26] R. W. Hendricks, "Space charge effects in proportional counters," Rev. Sci. Instrum., vol. 40, pp. 1216-1223, 1969.

[27] H. Sipila and V. Vanha-Honko, "The counting rate dependent gain shift and the space charge in proportional counters," Nucl. Instrum. Meth., vol. 153 , pp. $461-464,1978$.

[28] F. P. Santos, T. H. V. T. Dias, A. D. Stauffer and C. A. N. Conde, "Three-dimensional Monte Carlo calculation of the VUV electroluminescence and other electron transport parameters in xenon," J. Phys. D: Appl. Phys., vol. 27, pp. 42-48, 1994.

[29] A. Williams and R. I. Sara, "parameters affecting the resolution of a proportional counter," Int. J. Appl. Radiat. Isotopes, vol. 13, pp. 229-238, 1962. 